\title{
Hepatitis C: Virological Aspects and Practical Implications
}

\author{
Antonio Alci Barone \\ Laboratory of Hepatitis, LIM 47 DMIP-HC-FMUSP; São Paulo, SP, Brazil
}

The hepatitis C virus (HCV) is a member of the Hepacivirus genus of the family Flaviviridae.

Other important pathogens for humans, such as the dengue virus and the yellow fever virus, belong to this family, as do infectious agents in animals, such as the bovine viral diarrhea virus and the classical swine flu virus.

The shape of HCV is spherical (50 $\mathrm{nm}$ in diameter), and it has an enveloped nucleocapsid and a single-stranded RNA genome of positive polarity. The HCV genome, with approximately 10,000 nucleotides, consists of a single long open reading frame (ORF) flanked by two noncoding regions (NCRs) at the initial 5' end, at which the internal ribosome entry segment (IRES) is located, and at the 3' end. Its translation yields a large polyprotein (with nearly 3000 amino acids) that is processed by viral and host cell proteases into structural proteins, including the core, envelope 1 (E1), E2, and p7 proteins, as well as the nonstructural proteins NS2, NS3, NS4, and NS5. Recently, an alternate reading frame that codifies an F protein with more than 160 amino acids has been identified. However, its expression in natural HCV infection has not been confirmed.

Structural proteins are cleaved by enzymes of the parasitized cell. Envelope proteins are extensively glycosylated and are involved in the binding with receptors as well as in the entrance and fusion of the virus. The function of $\mathrm{p} 7$ protein remains unknown. Nonstructural proteins, initially NS2 and subsequently NS3, undergo self-cleavage and position themselves in transmembrane domains across the host cell membrane and into the cytosol or lumen (Figure 1).

Unlike that of the hepatitis B virus (HBV), the HCV genome does not invade the infected cell nucleus. After the binding through receptors (CD81, a tetraspanin, and the low density lipoprotein receptor), the $\mathrm{HCV}$ genome acts directly as an mRNA in the cytoplasm, where the translation is initiated through the IRES in the 5' NCR. The protein produced is subsequently processed by the cell enzymes and by the enzymes within the virus itself, yielding structural and nonstructural proteins. After synthesis and maturation, these nonstructural proteins and the RNA form replication complexes that combine with the membrane and catalyze the translation of intermediate negative strands of RNA, from which positivestrand progeny are generated. The genomic RNA and capsid proteins unite, forming the nucleocapsid, which is transported in cytoplasmic vesicles. While passing through the Golgi complex, these vesicles assemble with the other particles and undergo exocytosis and cell release (Figure 2).

The study of the HCV genome, even in samples obtained from a single individual, reveals great heterogeneity among the HCV genotypes. Genotypes in which multiple mutants

The Brazilian Journal of Infectious Diseases $\quad$ 2007;11 (5) Suppl. 1:12-13. (C) 2007 by The Brazilian Journal of Infectious Diseases and Contexto Publishing. All rights reserved. coexist have been designated quasispecies. The multiple mutations represent a rapid and very efficient mechanism for the virus to evade the immune response and persist in the host. The selection process and the process of adaptation to the host have led to the evolution to different HCV genotypes. The classification system most commonly used is that proposed by Simmonds et al. and is based on the similarity of the sequence of nucleotides using the following criteria: similarity lower than $72 \%$ characterizes a new genotype; similarity between 75 and $86 \%$ characterizes a new subgenotype. There are 6 genotypes, which are numbered from 1 to 6 , with subgenotypes $1 \mathrm{a}, 1 \mathrm{~b}, 1 \mathrm{c}, 2 \mathrm{a}, 2 \mathrm{~b}$, 2c, 3a, 3b, 4a, 5a, and 6a. Although the criterion is based on molecular biology, this classification has practical, pathogenetic, epidemiological, and treatment-related implications. Therefore, subgenotype 1a is more prevalent in the USA, 1b in Japan, 3a in Scotland, and 4a in Egypt/ Zaire. In Brazil, genotype 1 is found in approximately $60 \%$ of the patients, followed by genotype 3 , which is found in 20 to $30 \%$, and genotype 2 , which is found in a lower percentage. Subgenotype 1b can cause severe forms of the infection and, similar to genotype 4, does not respond as well to treatment with IFN- $\alpha$. Therefore, genotypes 1 and 4 should be treated for 48 weeks.

The important advances in the knowledge of viral hepatitis $\mathrm{B}$ and $\mathrm{C}$ are a consequence of some facts that will be discussed herein.

The Use of Chimpanzees as a Model for the Study of Viral Hepatitis

Although chimpanzees are not natural hosts for these viruses, they reproduce the disease, thus allowing important discoveries:

- Epidemiological studies: knowledge of these diseases as communicable;

- Infectivity and titers of pools of HCV and HBV, obtained from the infected animals;

- Infectivity of the molecular clones of $\mathrm{HCV}$ and importance of genetic elements specific to $\mathrm{HCV}$;

- Neutralization capacity of antibodies specific for HBV and HCV;

- Protective immunity, tested through re-exposure to the viruses;

- Mutants that escape to humoral and cellular immunity could be recognized.

However, the use of nonhuman primates has advantages and disadvantages.

\section{Advantages}

The only animal susceptible to the acute and chronic forms of the disease; non-selected population; and sequential biopsies. 
Figure 1. Organization of the HCV genome, polyprotein processing, and protein topology [1].

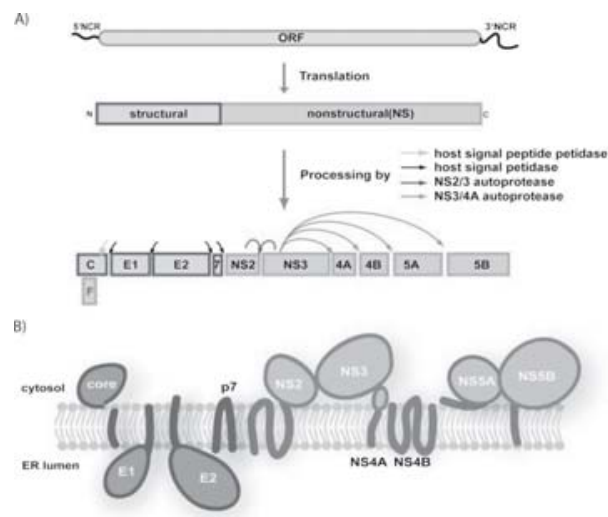

Figure 3. Systems used in the HCV in vitro study [1].

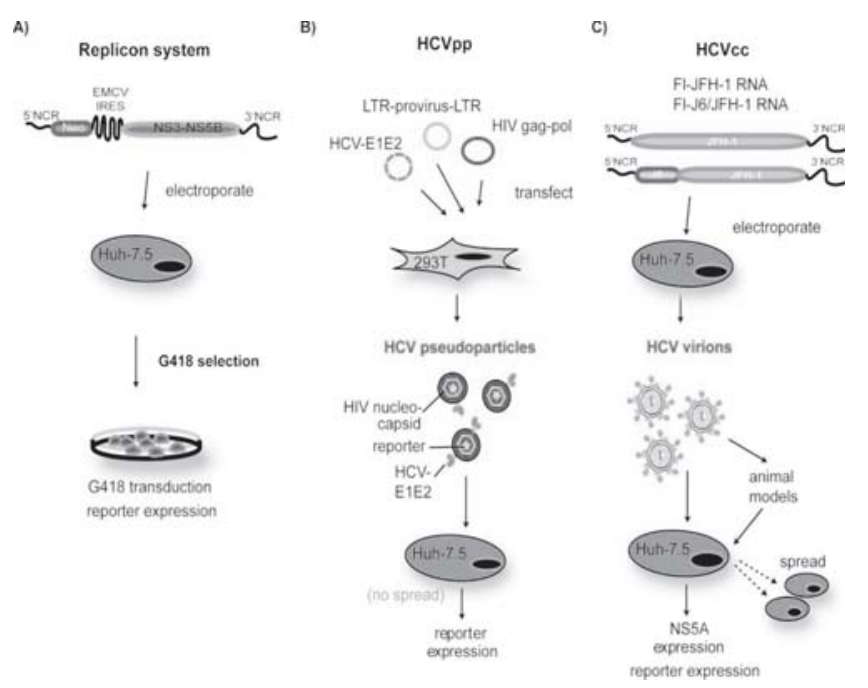

Disadvantages

Ethical considerations regarding the use of primates; cost and availability; rarity of vertical transmission; more benign disease presentation; and weaker, more limited immune response

\section{Use of Replicons}

In HCV, subgenomic RNA replicons are those in which the structural region has been replaced by the neomycin phosphotransferase gene, and the translation of the nonstructural proteins is regulated by the IRES of the encephalomyocarditis virus. Through this experimental approach, it became possible for the first time to effectively and efficiently replicate HCV in vitro in cultured Huh-7 human hepatoma cells. Interestingly, some simple amino acid substitutions can increase the replication efficiency by up to 10,000 times in all of the nonstructural proteins. The replicon system allowed the clarification of important aspects of the virus life cycle, as well as simplifying the evaluation of new antiviral strategies. However, some aspects of the virus life cycle cannot be studied using this system.
Figure 2. HCV cycle in the host cell [1].

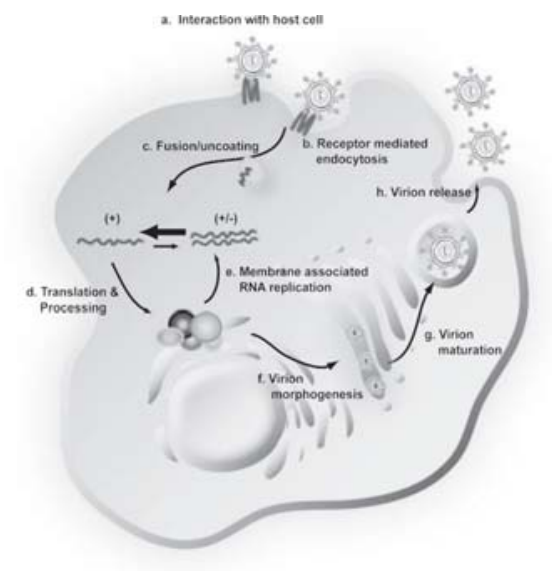

\section{Efficient Cell Culture Systems for HCV}

A 32-year-old male patient of Asian origin presented a profile consistent with fulminant hepatitis. Using reverse transcriptase polymerase chain reaction, HCV RNA was detected in the serum during the acute phase and not during the remission phase.

Using these samples, the complete HCV genome was recovered and cloned. This strain, designated JFH1, has 9678 bp in the genome, with a single long ORF (nt 341-9439), and encodes 3033 amino acids The transfection of this sample into HUH-7.5.1 cultured cells allowed three independent groups of researchers to obtain HCV infecting particles, for tissue culture as well as for chimpanzee. These studies [2-4] were published, between June and July of 2005, in the following journals: Proceedings of the National Academy of Sciences, Nature Medicine, and Science.

The infectious virions of HCV obtained in this way have been used to infect laboratory animals and naïve cells. Such infection can be monitored by the detection of the expression of the NS5A, through analysis of reporter genes, or by direct measurement of viral RNA (Figure 3).

In conclusion, more recent findings regarding the virological aspects of HCV have greatly increased the possibility of thoroughly studying this agent and especially its relationship with the human host. Based on this approach, it will be possible to better understand how to combat the virus in chronically infected patients, thus preventing the progression of the disease and its consequences.

\section{References}

1. Tellinghuissen T.L., et al. Studying Hepatitis C virus: making the best of a bad virus. Journal of Virology 2007;81(17):8853-67.

2. Barthenschlager B., et al. Efficient hepatitis C virus cell culture: what a difference the host cell makes. PNAS 2005;102(28):973940.

3. Wakita T., et al. Production of infectious hepatitis $\mathrm{C}$ virus in tissue culture from a cloned viral genome. Nature medicine 2005;11(7):791-6.

4. Lindenbach B.D., et al. Complete replication of hepatitis $C$ virus in cell culture. Science 2005;309:623-6.

5. Kato T., et al. Sequence analysis of Hepatitis C virus isolated from a fulminant hepatitis patient. J Med Virol 2001;64:334-9. 